\title{
Determining the physical limits on semi-active control performance: a tutorial
}

\author{
P. S. Harvey Jr. ${ }^{1, *, \dagger}$, H. P. Gavin ${ }^{1}$, J. T. Scruggs ${ }^{2}$ and J. M. Rinker ${ }^{1}$ \\ ${ }^{1}$ Civil \& Environmental Engineering, Duke University, Durham, North Carolina, USA \\ ${ }^{2}$ Civil \& Environmental Engineering, University of Michigan, Ann Arbor, Michigan, USA
}

\begin{abstract}
SUMMARY
Control forces in semi-active control systems are constrained by the dynamics of actuators that regulate energy transmission through variable damping and/or stiffness mechanisms. The potential benefit of the development and implementation of new semi-active control devices and applications can be determined by optimizing the controlled performance subject to the constraints of the dynamics of the system being controlled (given by the state equations), the constraints associated with the dynamics of the semi-active device, and the expected external forcing.

Performance optimization of semi-active control systems is a constrained two-point boundary value problem. This paper shows how this constrained problem can be transformed into an unconstrained problem, and how to easily solve the related unconstrained problem with MATLAB. The method is illustrated on the performance optimization of a simple semi-active tuned-mass-damper for a structure subjected to ground accelerations. Several possible extensions of this method and application are offered. Copyright @ 2013 John Wiley \& Sons, Ltd.
\end{abstract}

Received 6 September 2012; Revised 19 July 2013; Accepted 23 July 2013

KEY WORDS: $\quad$ semi-active; open-loop; constrained control; two-point boundary value problem; tuned-mass-damper

\section{INTRODUCTION}

Over the last several decades, a large number of semi-active control devices have been developed for a broad range of applications. Since the publication of review articles on semi-active control [1,2], research has progressed on semi-active stiffness devices [3-7], semi-active damping devices [8-10], and semi-active friction devices [11,12]. Models for these devices involve constraints, either directly on the device forces or indirectly on an internal variable such as a valve position, a solenoid voltage, or an electrical resistance.

There are two principle advantages of implementing semi-active control. The first is that the power that a semi-active device is able to regulate within the structure can be orders of magnitude greater than the power required to regulate the device properties (e.g., damping and/or stiffness). The second is that the controlled system is unconditionally stable in a bounded-input, bounded-output sense regardless of the feedback law implemented. A potential disadvantage of semi-active control systems is that, for some applications, closed-loop semi-active performance may be only marginally better than that of simpler passive control systems [1]. Additionally, actuation constraints of semiactive control systems render the system nonlinear and performance can be assessed and optimized only through transient response simulations. It is therefore common practice to evaluate the performance of semi-actively controlled systems for a particular feedback law and compare the result with a passively controlled system. This method of performance evaluation may be used to assess the potential

*Correspondence to: P. S. Harvey Jr., Duke University, Civil \& Environmental Engineering, Durham, North Carolina, USA.

†E-mail: Philip.Harvey@ Duke.edu 
performance of a semi-active control system subject to the constraint of the feedback control rule, which can be overly restrictive.

A more complete evaluation of the potential benefit of a semi-active control system eliminates the restriction of the feedback control rule. Methods of trajectory optimization may be used to determine the best possible performance, as defined by a particular objective function, while strictly adhering to the constraints of a particular semi-active device, the structural system into which it is applied, and the external forcing. This optimization enables a rigorous evaluation of new semi-active devices and a meaningful comparison with existing semi-active devices and passive devices. Semi-active devices that can achieve performance levels sufficiently better than those of existing passive devices or alternative semi-active devices merit the development of control hardware and feedback control rules.

The correct formulation, and importance, of constrained control problems have been known for decades. Kirk emphasizes that 'the optimal [constrained] control history ... cannot be determined, in general, by calculating the optimal [unconstrained] control history and allowing it to saturate whenever the stipulated boundaries are violated' [13] (p. 236). Further, Tseng and Hedrick prove that 'clippedoptimal is sub-optimal in the sense that it minimizes only the instantaneous performance index difference [and] does not guarantee optimality in minimizing [an integral] performance index' [14] (p. 556).

We note here that in a dynamical control system, $\dot{\boldsymbol{x}}=\boldsymbol{f}(\boldsymbol{x}, u ; t)$, changing the controls $u$ at time $t$ changes $\dot{\boldsymbol{x}}$ (but not $\boldsymbol{x}$ ) at time $t$. So any state-dependent performance index cannot be instantaneously improved by changing the controls at time $t$. It is therefore rational to minimize integral cost functions. The choice of the objective function is subjective, and reflects the control engineer's best judgment regarding the purpose of the control system.

Following the work of Tseng and Hedrick [14], this short paper states the semi-active control optimization problem as a constrained two-point boundary value problem (TPBVP), and gives a solution procedure by which the constraints are eliminated, reducing the problem to an unconstrained TPBVP. The method is illustrated on a simplified tuned-mass-damper (TMD) with a supplemental semi-active damper in which the dynamics are linear (except for the actuation constraints) and the Lagrangian of the cost function is quadratic. The purpose of this paper is to provide a concise tutorial on semi-active performance optimization that illustrates, in detail, just how easy it is to setup and solve such problems.

The method for optimizing semi-active control trajectories illustrated in this paper is fundamentally distinct from feedback control methods such as clipped optimal or Lyapunov-based controls. The motivation for the use of such techniques is for the design of real-time feedback laws that are straight-forward to compute in closed form, easy to implement, and feasible under semi-active constraints. However, such approaches are always suboptimal; that is, they leave open the possibility that there may be some other feedback law that, if implemented, could have led to a much more favorable dynamic response. Indeed, irrespective of the measure of optimality chosen, the optimal feedback problem for semi-actively constrained systems remains an open problem.

By contrast, techniques exist for the rigorous computation of the optimal physically-achievable performance of a semi-actively constrained system, given precise models of the system and disturbance. Such techniques do not result in an optimal feedback law, and the optimized control inputs are anticipatory; that is, they presume knowledge of the entire disturbance trajectory. These solutions are highly valuable in the context of semi-active control system analysis, for several reasons:

- First, they can be used to assess the ultimate viability of semi-active control for a given application. More specifically, they determine whether a given semi-active device, embedded within a given structure and subjected to a given disturbance, can possibly achieve a level of performance the designer requires. This effectively enables the hardware of an application to be assessed prior to the design of a feedback law. This observation was a significant motivation for investigations in this area in automotive suspensions applications, by Hrovat et al. [15], as well as, Tseng and Hedrick [14].

- Second, the nature of the optimized trajectory for the control input can be used to gain significant intuition regarding the mathematical structure a semi-active feedback law should have. For example, in [16], Leavitt et al. used optimal control techniques to infer heuristic switching rules for a semi-active variable-stiffness device under harmonic excitation. Likewise, in [17], Harvey et al. examined optimal control trajectories for a semi-actively controlled isolation system 
subjected to a near-field earthquake pulse record and showed that the qualitative nature of the optimized semi-active force depends on the specific parameters of the pulse record as well as the performance measure. For certain parameter combinations, it resembles a 'pseudo-negative stiffness' damping force. For others, it is more reminiscent of nonlinear viscous damping.

- Third, the optimal control techniques discussed in this paper are directly applicable to model predictive control synthesis [18]. This is a feedback control technique in which the optimal control trajectory is periodically solved in real time from the present time over a receding horizon, using an iteratively-updated forecast of the future disturbance trajectory. Such techniques have recently been investigated for semi-active applications by Giorgetti et al. [19].

Section 2 of this paper rigorously defines semi-active optimization as a constrained TPBVP, provides optimality conditions, and presents a solution methodology for this class of problems. In section 3, the performance of an optimal semi-active control system is illustrated in the context of a semi-active TMD application, and is compared with performance achieved via clipped linearquadratic regulator (LQR).

The primary objective of this paper is to provide a tutorial on how to solve the optimal semi-active control problem and to illustrate the method with a simple example. As such, emphasis is placed on the application to a relatively simple system and on providing detailed information on how the method can be implemented. MATLAB [20] code is provided in the Appendix.

\section{PROBLEM STATEMENT}

An admissible scalar control trajectory $u(t)$ is to be applied to a non-autonomous system

$$
\dot{\boldsymbol{x}}(t)=\boldsymbol{A} \boldsymbol{x}(t)+\boldsymbol{B} u(t)+\boldsymbol{B}_{w} w(t), \quad \boldsymbol{x}(0)=\boldsymbol{x}_{0}, \quad \boldsymbol{x}(t) \in \mathbb{R}^{n}
$$

in order to minimize the following cost functional of the states $\boldsymbol{x}(t)$ and control input $u(t)$ :

$$
J=\int_{0}^{t_{\mathrm{f}}} L(\boldsymbol{x}, u ; t) d t \equiv \int_{0}^{t_{\mathrm{f}}} \frac{1}{2}\left[\begin{array}{c}
\boldsymbol{x}(t) \\
u(t)
\end{array}\right]^{\top}\left[\begin{array}{cc}
\boldsymbol{Q} & \boldsymbol{S} \\
\boldsymbol{S}^{\top} & R
\end{array}\right]\left[\begin{array}{l}
\boldsymbol{x}(t) \\
u(t)
\end{array}\right] d t .
$$

The linear, time-invariant plant (1) is parameterized as follows: $\boldsymbol{A} \in \mathbb{R}^{n \times n}$ is the dynamics matrix, $\boldsymbol{B} \in \mathbb{R}^{n}$ is the control input matrix, and $\boldsymbol{B}_{w} \in \mathbb{R}^{n}$ is the input matrix associated with the known, deterministic exogenous disturbance $w(t)$. The Lagrangian $L(\cdot)$ is quadratic with state weighting matrix $\boldsymbol{Q} \in \mathbb{R}^{n \times n}$, control weighting scalar $R$, and bilinear state-control weighting matrix $S \in \mathbb{R}^{n}$.

Typically, controllable dampers have performance limitations described by a maximum achievable control force amplitude $u_{\max }$, a maximum achievable damping coefficient $c_{\max }$, and a dissipative force-velocity relation. So, for semi-active damping, feasible control forces are bounded by

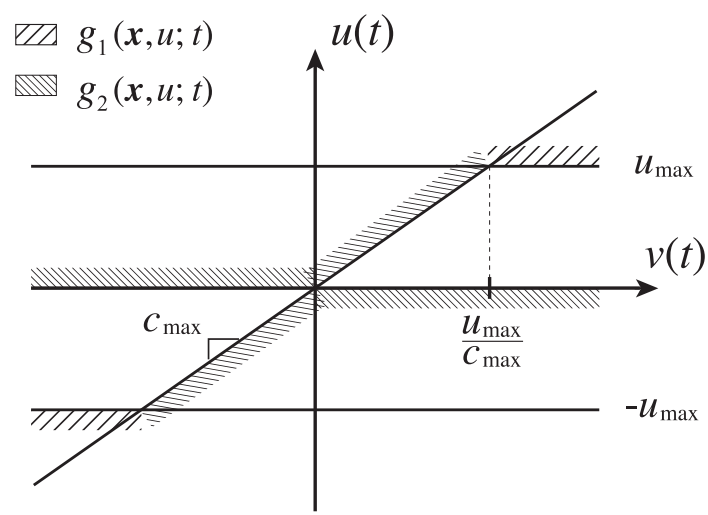

Figure 1. Sector-bound constraint for semi-active damping device. $v(t)$ is the velocity across the actuator. 
sectors shown in Figure 1, where $v(t)$ is the velocity across the actuator. The former limitation implies $|u(t)|<u_{\max }$ and the latter implies $|u(t)| \in\left[0, c_{\max }|v(t)|\right]$; such constraints may be expressed by the following nonlinear inequality constraint equations:

$$
\boldsymbol{g}(\boldsymbol{x}, u ; t)=\left[\begin{array}{c}
u^{2}(t)-u_{\max }^{2} \\
u(t)\left(u(t)-c_{\max } \boldsymbol{T}^{\top} \boldsymbol{x}(t)\right)
\end{array}\right] \leqslant \mathbf{0}
$$

where the transformation vector $\boldsymbol{T} \in \mathbb{R}^{n}$ extracts the velocity across the actuator, $v(t)=\boldsymbol{T}^{\top} \boldsymbol{x}(t)$. For other semi-active device models, the feasible region may take other forms, as described in Section 5 .

The minimization of $J$ is subject to the equality constraint (1) and the inequality constraint (3). The Hamiltonian is therefore defined as

$$
H(\boldsymbol{x}, u, \boldsymbol{p}, \lambda ; t) \equiv L(\boldsymbol{x}, u ; t)+\boldsymbol{p}^{\top}(t)\left[\boldsymbol{A} \boldsymbol{x}(t)+\boldsymbol{B} u(t)+\boldsymbol{B}_{w} w(t)\right]+\lambda^{\top}(t) \boldsymbol{g}(\boldsymbol{x}, u ; t)
$$

where $\boldsymbol{p}(t) \in \mathbb{R}^{n}$ is a Lagrange multiplier vector (or costate) for the dynamic constraint (1), and $\lambda(t) \in \mathbb{R}^{2}$ are the Lagrange multipliers for the inequality constraint (3). Note that all $\lambda_{i}(t) \geqslant 0$. In the usual way [13], adjoining the constraints with multipliers to the performance index $J$, we have

$$
J_{A}=\int_{0}^{t_{\mathrm{f}}}\left[H(\boldsymbol{x}, u, \boldsymbol{p}, \lambda ; t)-\boldsymbol{p}^{\top}(t) \dot{\boldsymbol{x}}(t)\right] d t .
$$

Following the calculus of variations, the first-order necessary conditions for optimality are [13]

$$
\begin{gathered}
\dot{\boldsymbol{x}}(t)=\frac{\partial H}{\partial \boldsymbol{p}}=\boldsymbol{A} \boldsymbol{x}(t)+\boldsymbol{B} u(t)+\boldsymbol{B}_{w} w(t), \quad \boldsymbol{x}(0)=\boldsymbol{x}_{0} \\
\dot{\boldsymbol{p}}(t)=-\frac{\partial H}{\partial \boldsymbol{x}}=-\boldsymbol{Q} \boldsymbol{x}(t)-\boldsymbol{S} u(t)-\boldsymbol{A}^{\top} \boldsymbol{p}(t)+c_{\text {max }} u(t) \lambda_{2}(t) \boldsymbol{T}, \quad \boldsymbol{p}\left(t_{\mathrm{f}}\right)=\mathbf{0} \\
0=\frac{\partial H}{\partial u}=\boldsymbol{S}^{\top} \boldsymbol{x}(t)+R u(t)+\boldsymbol{B}^{\top} \boldsymbol{p}(t)+2 u(t) \lambda_{1}(t)+\left(2 u(t)-c_{\max } \boldsymbol{T}^{\top} \boldsymbol{x}(t)\right) \lambda_{2}(t) \\
\mathbf{0} \geqslant \frac{\partial H}{\partial \boldsymbol{\lambda}}=\boldsymbol{g}(\boldsymbol{x}, u ; t) .
\end{gathered}
$$

Equation (6) constitutes a differential-algebraic TPBVP. The following section gives the solution procedure proposed by Harvey et al. [17], which is an extension of the work of Tseng and Hedrick [14].

\subsection{Solution procedure}

To solve the necessary conditions (6), the following quadratic program is solved at each time $t$ :

$$
\min _{u(t)} \max _{\lambda(t) \geq \mathbf{0}} H(\boldsymbol{x}, u, \boldsymbol{p}, \lambda ; t) .
$$

The unconstrained optimal control is given by the stationarity condition (6c) for $\boldsymbol{\lambda}=\mathbf{0}$.

$$
u_{\text {active }}(t)=-R^{-1}\left(\boldsymbol{S}^{\top} \boldsymbol{x}(t)+\boldsymbol{B}^{\top} \boldsymbol{p}(t)\right) .
$$

The subscript 'active' is used here to represent the finite-horizon unconstrained optimal control input, not an LQR or linear-quadratic-Gaussian feedback controller. Then, using the following saturation function to ensure feasibility,

$$
u_{\text {sat }}(t)=\operatorname{sat}\left(\boldsymbol{x}, u_{\text {active }} ; t\right)= \begin{cases}u_{\text {active }}: & \boldsymbol{g}\left(\boldsymbol{x}, u_{\text {active }} ; t\right) \leqslant \mathbf{0} \\ \arg _{u(t)}\left\{g_{j}(\boldsymbol{x}, u ; t)=0\right\}: & g_{j}\left(\boldsymbol{x}, u_{\text {active }} ; t\right)>0\end{cases}
$$

At times where the active control input is infeasible, we saturate $u(t)$ to the constraint boundary, $g_{j}(\boldsymbol{x}, u ; t)=0$, and the $j$ th Lagrange multiplier $\lambda_{j}(t)$ is determined from (6c) such that the Hamiltonian 
has a saddle point at the constraint boundary. If two or more constraints are violated, we saturate to the most restrictive constraint.

\subsection{Saturation function for semi-active damper}

For the semi-active damper constraint (3), the saturation function (9) can be implemented numerically as follows [17]:

1. Calculate $\boldsymbol{g}\left(\boldsymbol{x}, u_{\text {active }} ; t\right)$ and the velocity across the actuator $v(t)=\boldsymbol{T}^{\top} \boldsymbol{x}(t)$.

2. Perform the following checks:

(a) if $\boldsymbol{g}\left(\boldsymbol{x}, u_{\text {active }} ; t\right) \leqslant \mathbf{0}$, set $u_{\text {sat }}(t)=u_{\text {active }}(t), \lambda_{1}(t)=0, \lambda_{2}(t)=0$, and break;

(b) if $u_{\text {active }}(t) v(t)<0$, set $u_{\text {sat }}(t)=0, \lambda_{1}(t)=0$,

$$
\lambda_{2}(t)=\left(\boldsymbol{S}^{\top} \boldsymbol{x}(t)+\boldsymbol{B}^{\top} \boldsymbol{p}(t)\right) /\left(c_{\max } v(t)\right),
$$

and break;

(c) if $|v(t)|>u_{\max } / c_{\max }$, set $u_{\text {sat }}(t)=u_{\max } \operatorname{sign}\left(u_{\text {active }}(t)\right)$ where $\operatorname{sign}(\cdot)$ is the signum function,

$$
\lambda_{1}(t)=-\left(\boldsymbol{S}^{\top} \boldsymbol{x}(t)+R u_{\mathrm{sat}}(t)+\boldsymbol{B}^{\top} \boldsymbol{p}(t)\right) /\left(2 u_{\mathrm{sat}}(t)\right),
$$

$\lambda_{2}(t)=0$, and break;

(d) if $v(t)=0$, set $u_{\text {sat }}(t)=0, \lambda_{1}(t)=0, \lambda_{2}(t)=0$, and break;

(e) otherwise, set $u_{\text {sat }}(t)=c_{\max } v(t), \lambda_{1}(t)=0$,

$$
\lambda_{2}(t)=-\left(\boldsymbol{S}^{\top} \boldsymbol{x}(t)+R u_{\mathrm{sat}}(t)+\boldsymbol{B}^{\top} \boldsymbol{p}(t)\right) /\left(c_{\max } v(t)\right),
$$

and break.

Note that step 2(d) is in place to handle the singularity in (6c) at $v(t)=0$; that is, for $v(t)=0$, the equality constraint $u(t)=0$ must be satisfied, thus making $\lambda_{2}(t)$ arbitrary, for which we have chosen $\lambda_{2}(t)=0$. In MATLAB, the saturation function can be implemented by calling a function such as sat $(x$, uactive, $p)$ given in Appendix A.

In solving for $u_{\text {sat }}(t)$ and $\lambda(t)$ and substituting them into equations (6a) and (6b), the TPBVP is now unconstrained, as given by

$$
\frac{d}{d t}\left[\begin{array}{l}
\boldsymbol{x}(t) \\
\boldsymbol{p}(t)
\end{array}\right]=\left[\begin{array}{cc}
\boldsymbol{A} & \mathbf{0} \\
-\boldsymbol{Q} & -\boldsymbol{A}^{\top}
\end{array}\right]\left[\begin{array}{l}
\boldsymbol{x}(t) \\
\boldsymbol{p}(t)
\end{array}\right]+\left[\begin{array}{c}
\boldsymbol{B} \\
-\boldsymbol{S}+c_{\max } \lambda_{2}(t) \boldsymbol{T}
\end{array}\right] u_{\mathrm{sat}}(t)+\left[\begin{array}{c}
\boldsymbol{B}_{w} \\
\mathbf{0}
\end{array}\right] w(t)
$$

with boundary conditions

$$
\left[\begin{array}{l}
\boldsymbol{x}(0) \\
\boldsymbol{p}\left(t_{\mathrm{f}}\right)
\end{array}\right]=\left[\begin{array}{c}
\boldsymbol{x}_{0} \\
\mathbf{0}
\end{array}\right]
$$

To ensure that the necessary conditions (6) are satisfied, the states $\boldsymbol{x}(t)$ and costates $\boldsymbol{p}(t)$ must be determined by numerically solving (13). Numerical methods to solve unconstrained TPBVPs are well established, for example, shooting methods, finite differences, and finite elements. In this study, the unconstrained TPBVP is solved with the MATLAB function bvp $4 \mathrm{c} . \mathrm{m}$, which implements a collocation method with piecewise cubic interpolation satisfying the boundary conditions over each time step [21]. The following section gives a numerical demonstration of how to implement bvp $4 \mathrm{c}$ to solve (13) and determine optimal control trajectories that adhere to semi-active constraints. 


\section{NUMERICAL EXAMPLE}

\subsection{Tuned-mass-damper system}

To illustrate the performance optimization of a semi-active system, the semi-active performance of a simple semi-active TMD model is optimized to suppress seismic responses. The model is very similar to the system studied by Hrovat et al. [22], except that in this study, the system is subjected to base acceleration $\ddot{x}_{\mathrm{g}}$, as shown in Figure 2. The mass-normalized equations of motion, which model the vibration of the system, are

$$
\begin{gathered}
(1+\mu) \ddot{x}_{\mathrm{s}}(t)+\mu \ddot{x}_{\mathrm{t}}(t)+2 \zeta_{\mathrm{s}} \omega_{\mathrm{s}} \dot{x}_{\mathrm{s}}(t)+\omega_{\mathrm{s}}^{2} x_{\mathrm{s}}(t)=-(1+\mu) \ddot{x}_{\mathrm{g}}(t) \\
\ddot{x}_{\mathrm{s}}(t)+\ddot{x}_{\mathrm{t}}(t)+2 \zeta_{\mathrm{t}} \omega_{\mathrm{t}} \dot{x}_{\mathrm{t}}(t)+\omega_{\mathrm{t}}^{2} x_{\mathrm{t}}(t)+u(t) / m_{\mathrm{t}}=-\ddot{x}_{\mathrm{g}}(t)
\end{gathered}
$$

with the following parameters defined [22]:

$$
\mu=m_{\mathrm{t}} / m_{\mathrm{s}}, \quad \omega_{\mathrm{s}}=\sqrt{k_{\mathrm{s}} / m_{\mathrm{s}}}, \quad \omega_{\mathrm{t}}=\sqrt{k_{\mathrm{t}} / m_{\mathrm{t}}}, \quad \zeta_{\mathrm{s}}=c_{\mathrm{s}} /\left(2 m_{\mathrm{s}} \omega_{\mathrm{s}}\right), \quad \zeta_{\mathrm{t}}=c_{\mathrm{t}} /\left(2 m_{\mathrm{t}} \omega_{\mathrm{t}}\right) .
$$

Equation (15) can be represented in state-space form (1) where $\boldsymbol{x}(t)=\left[x_{\mathrm{s}}(t), x_{\mathrm{t}}(t), \dot{x}_{\mathrm{s}}(t), \dot{x}_{\mathrm{t}}(t)\right]^{\top} \in \mathbb{R}^{4}$, $w(t)=\ddot{x}_{\mathrm{g}}(t)$,

$$
\boldsymbol{A}=\left[\begin{array}{cccc}
0 & 0 & 1 & 0 \\
0 & 0 & 0 & 1 \\
-\omega_{\mathrm{s}}^{2} & \mu \omega_{\mathrm{t}}^{2} & -2 \zeta_{\mathrm{s}} \omega_{\mathrm{s}} & \mu 2 \zeta_{\mathrm{t}} \omega_{\mathrm{t}} \\
\omega_{\mathrm{s}}^{2} & -(1+\mu) \omega_{\mathrm{t}}^{2} & 2 \zeta_{\mathrm{s}} \omega_{\mathrm{s}} & -(1+\mu) 2 \zeta_{\mathrm{t}} \omega_{\mathrm{t}}
\end{array}\right], \boldsymbol{B}=\left[\begin{array}{c}
0 \\
0 \\
\mu / m_{\mathrm{t}} \\
-(1+\mu) / m_{\mathrm{t}}
\end{array}\right], \boldsymbol{B}_{w}=\left[\begin{array}{c}
0 \\
0 \\
-1 \\
0
\end{array}\right]
$$

The mass ratio $\mu=0.10$ and the TMD natural frequency $\omega_{\mathrm{t}}$ is the optimum tuning frequency $\omega_{\mathrm{t}}^{*}$ discussed in Section 3.1.1. Table I gives numerical values for the system parameters. As a benchmark for comparison, the optimal performance will be compared with the following three cases.

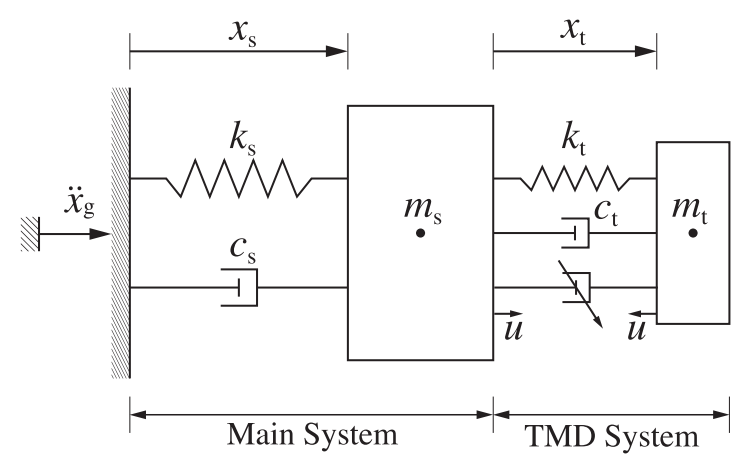

Figure 2. Structural model of a single-degree-of-freedom structure with tuned-mass-damper (TMD).

Table I. Parameter values used in simulation.

\begin{tabular}{lll}
\hline Building data & Semi-active TMD data & Passive TMD data [22] \\
\hline$m_{\mathrm{s}}=1 \times 10^{6} \mathrm{~kg}$ & $m_{\mathrm{t}}=1 \times 10^{5} \mathrm{~kg}$ & $m_{\mathrm{t}}=1 \times 10^{5} \mathrm{~kg}$ \\
$k_{\mathrm{s}}=1 \times 10^{6} \mathrm{~N} / \mathrm{m}$ & $k_{\mathrm{t}}^{*}=8.26 \times 10^{4} \mathrm{~N} / \mathrm{m}$ & $k_{\mathrm{t}}^{*}=8.26 \times 10^{4} \mathrm{~N} / \mathrm{m}$ \\
$c_{\mathrm{s}}=2 \times 10^{4} \mathrm{~N} \mathrm{~s} / \mathrm{m}$ & $c_{\mathrm{t}}=1.82 \times 10^{3} \mathrm{~N} \mathrm{~s} / \mathrm{m}$ & $c_{\mathrm{t}}^{*}=3.36 \times 10^{4} \mathrm{~N} \mathrm{~s} / \mathrm{m}$ \\
$\zeta_{\mathrm{s}}=0.01$ & $\zeta_{\mathrm{t}}=0.01$ & $\zeta_{\mathrm{t}}^{*}=0.185$ \\
$\omega_{\mathrm{s}}=1.0 \mathrm{rad} / \mathrm{s}$ & $\omega_{\mathrm{t}}^{*}=0.909 \mathrm{rad} / \mathrm{s}$ & $\omega_{\mathrm{t}}^{*}=0.909 \mathrm{rad} / \mathrm{s}$ \\
\hline
\end{tabular}


3.1.1. Optimized passive tuned-mass-damper. A passive TMD with optimized parameters is used as the first benchmark for comparison. Parameter optimization of the passive TMD results in the following expressions for $\omega_{\mathrm{t}}^{*}$ and $\zeta_{\mathrm{t}}^{*}$ [23]:

$$
\begin{gathered}
\text { optimum tuning frequency: } \omega_{\mathrm{t}}^{*}=\frac{\omega_{\mathrm{s}}}{1+\mu}, \\
\text { optimum passive damping ratio : } \zeta_{\mathrm{t}}^{*}=\sqrt{\frac{3 \mu}{8(1+\mu)}} .
\end{gathered}
$$

Table I gives numerical values for the passive TMD system parameters. Note the passive damping force, $c_{\mathrm{t}}^{*} \boldsymbol{T}^{\top} \boldsymbol{x}(t)$, is to be clipped at the same level $u_{\max }$ as the semi-active device to ensure a fair comparison.

3.1.2. Clipped linear-quadratic regulator. The second control scheme-clipped LQR-is a somewhat ad hoc yet prevalent suboptimal scheme, based on LQR theory. Define the linear feedback control $u_{\mathrm{LQR}}=-R^{-1}(\boldsymbol{P B}+\boldsymbol{S})^{\top} \boldsymbol{x}$, where $\boldsymbol{P}$ is found by solving the algebraic Riccati equation

$$
\mathbf{0}=\boldsymbol{A}^{\top} \boldsymbol{P}+\boldsymbol{P A}-(\boldsymbol{P B}+\boldsymbol{S}) R^{-1}(\boldsymbol{P B}+\boldsymbol{S})^{\top}+\boldsymbol{Q} .
$$

In order to directly implement $u_{\mathrm{LQR}}$ in the compliant damper model, the feedback controls are clipped when the prescribed forces is infeasible.

3.1.3. Uncontrolled. Finally, the performance of the optimal control trajectory is juxtaposed against an uncontrolled structure with no TMD, which is essentially the response of a single-degree-of-freedom system with parameters given in the first column of Table I.

\subsection{Pulse-like disturbance model}

Two types of ground motions are considered in this study: an idealized pulse and a historical ground motion record. Analytical pulse models are useful in the systematic design and assessment of seismic protective systems. Furthermore, because of the smooth nature of the disturbance and responses, simulations are less computationally expensive, which can accelerate parameter tuning, for example, determining weighting matrices $\boldsymbol{Q}, R$ (or $\boldsymbol{R}$ for multiple control inputs), and $\boldsymbol{S}$.

The pulse acceleration in this study is given by [17]

$$
\ddot{x}_{\mathrm{g}}(t)=\left(\frac{t-t_{0}}{\tau}\right)^{\eta} \exp \left(-\frac{t-t_{0}}{\tau}\right) \cos \left(\omega_{\mathrm{p}}\left(t-t_{0}\right)-\varphi\right) .
$$

Accelerations are zero for $t<t_{0}$ and have a predominant period $T_{\mathrm{p}}=2 \pi / \omega_{\mathrm{p}}$. In order for the record to contain $N$ cycles of strong motion, the decay time constant $\tau$ is set to $N T_{\mathrm{p}} / 4$. Ground acceleration records should have negligibly small velocity and small displacement at the end of the record. For a zero terminal velocity, the phase constant $\varphi$ should be [17]

$$
\tan \varphi=\frac{3\left(\tau \omega_{\mathrm{p}}\right)^{2}-1}{3\left(\tau \omega_{\mathrm{p}}\right)-\left(\tau \omega_{\mathrm{p}}\right)^{3}} .
$$

To enforce small residual displacements, the second derivative of a scaled logistic is iteratively subtracted from the acceleration record until the displacement at the end of the record is close to zero. The associated fixed-point map is

$$
\ddot{x}_{\mathrm{g}}(t) \leftarrow \ddot{x}_{\mathrm{g}}(t)-x_{\mathrm{g}}\left(t_{\mathrm{f}}\right) \exp (-s)[1+\exp (-s)]^{3}[\exp (-s)-1] /(\tau / 2)^{2},
$$

where $x_{\mathrm{g}}\left(t_{\mathrm{f}}\right)$ is the displacement at the end of the record, and $s$ is a scaled time variable equal to $\left(t-t_{0}-\eta \tau\right) /(\tau / 2)$. In applying the fixed-point-map of equation (22), accelerations should not be 
reset to zero for $t<t_{0}$. For $\eta=2,1<N<5$, and $0.5<T_{\mathrm{p}}<4 \mathrm{~s}$, peak velocities scale with $T_{\mathrm{p}}$ and are approximately given by

$$
\dot{x}_{\mathrm{g}}^{\max }=\max \left[4.063 N^{-2.165} \exp (-4.403 / N), 2.329 N^{-1.336} \exp (-5.693 / N)\right] T_{\mathrm{p}} \pm 0.5 \% .
$$

$\mathrm{He}$ and Agrawal [24] validated a similar pulse model through comparison with numerous ground motions, corresponding response spectra, and the performance of passive energy dissipation systems. The pulse model used in the present work has a terminal velocity of zero (from equation (21)) and a terminal displacement of zero (from equation (22)). In this study, disturbance waveforms were scaled to match prescribed peak velocity values $V_{\mathrm{p}}$ by scaling accelerations by a factor of $V_{\mathrm{p}} / \dot{x}_{\mathrm{g}}^{\max }$. Figure 3(a) illustrates a sample disturbance record using the following disturbance parameters: $\omega_{\mathrm{p}}=1.0 \mathrm{rad} / \mathrm{s}, V_{\mathrm{p}}=0.8 \mathrm{~m} / \mathrm{s}, t_{0}=2.0 \mathrm{~s}, \eta=2$, and $N=2$.

\subsection{Performance index and numerical values}

In this example, the Lagrangian $L(\cdot)$ is selected as the square of the total acceleration of the primary structure:

$$
L(\boldsymbol{x}, u ; t)=\frac{1}{2}\left[\ddot{x}_{\mathrm{g}}(t)+\ddot{x}_{\mathrm{s}}(t)\right]^{2} \equiv \frac{1}{2}\left[\boldsymbol{A}_{(3,:)} \boldsymbol{x}(t)+\boldsymbol{B}_{(3)} u(t)\right]^{2}
$$

where $\boldsymbol{A}_{(3,:)}$ is the third row of the dynamics matrix, and $\boldsymbol{B}_{(3)}$ is the third entry of the control input vector. The state, control input, and cross weighting matrices are thus $\boldsymbol{Q}=\boldsymbol{A}_{(3,:)}^{\top} \boldsymbol{A}_{(3,:)}, R=\boldsymbol{B}_{(3)}^{\top} \boldsymbol{B}_{(3)}$, and $\boldsymbol{S}=\boldsymbol{A}_{(3,:)}^{\top} \boldsymbol{B}_{(3)}$.

We consider only adjustable control forces $u(t)$ that are constrained by (3). For the constraint $g_{1}(\boldsymbol{x}, u ; t)$, the maximum semi-active force $u_{\max }=5 \times 10^{4} \mathrm{~N}$ is used in simulation. For the maximum dissipating constraint $g_{2}(\boldsymbol{x}, u ; t)$, the velocity across the actuator is $\dot{x}_{\mathrm{t}}=\boldsymbol{T}^{\top} \boldsymbol{x}$ for which $\boldsymbol{T}^{\top}=\left[\begin{array}{llll}0 & 0 & 0 & 1\end{array}\right]$. The maximum damping coefficient is taken to be $c_{\max }=2 \zeta_{\max } \omega_{\mathrm{t}} m_{\mathrm{t}}$, with $\zeta_{\max }=18 \%$.

\subsection{MATLAB procedure}

Appendix A gives sample code for this example. The procedure involves first initializing the model parameters (line 2-3). The variables $d t$ and $\mathrm{nT}$ are the time step and length of the time vector
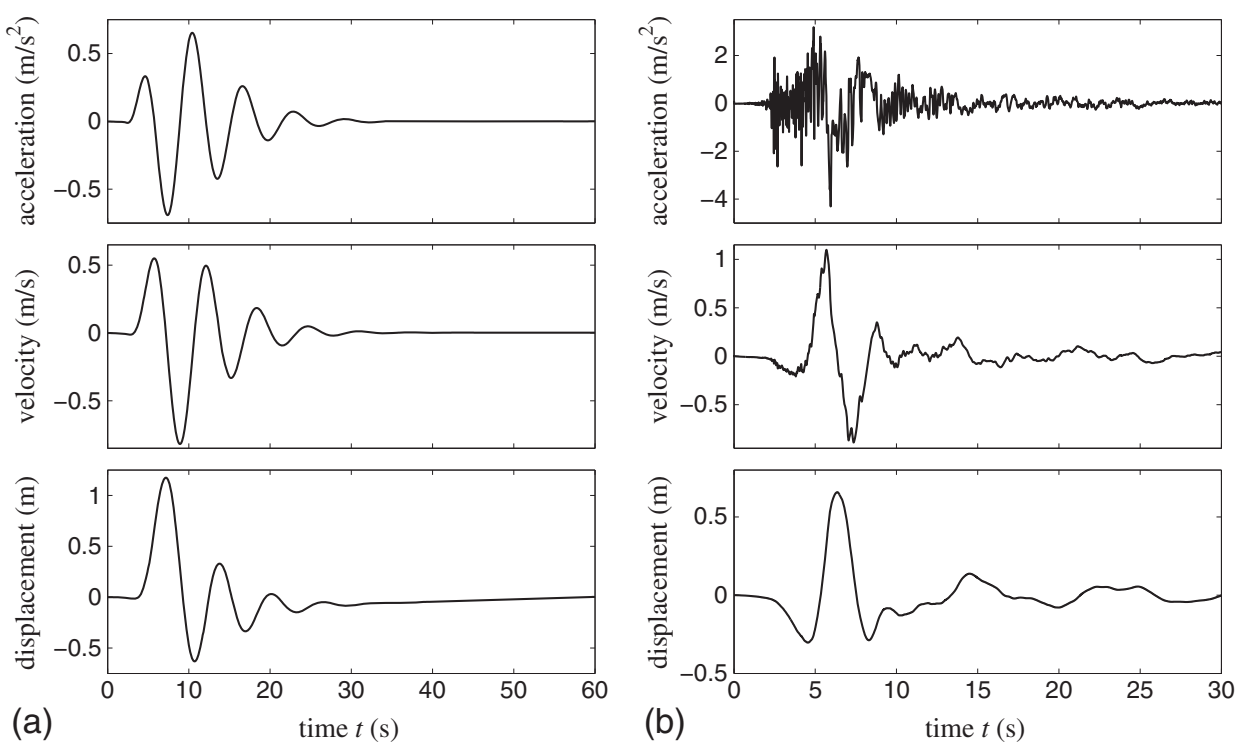

Figure 3. Acceleration, velocity, and displacement time histories of a pulse-like ground motion and a recorded ground motion. (a) Pulse-like ground motion for parameter values given in Section 3.2 and (b) 1979 Imperial Valley earthquake, El Centro Array \#6-230. 
$t$, respectively, used to linearly interpolate the disturbance history $w$ at intermediate times. The initial states $\times 0$ and terminal costates $\mathrm{pf}$ must also be specified. Concatenating the state and costate into a single vector, define $z=\varangle ; p]$. Specify global variables (line 1 ), which are accessed by the ordinary differential equation (ODE) function $z \operatorname{dot}(t, z, w)$, the boundary condition (BC) function bcfun $(z 0, z f, x 0, p f)$, and the saturation function sat ( $x$, uactive, $p)$.

In line 6 , the options are specified using bvpset. The maximum mesh discretization (the maximum number of time steps) NMax is increased to avoid premature termination of bvp $4 \mathrm{c}$; because bvp $4 \mathrm{c}$ uses an adaptive mesh, with NMax too small the evaluation may be terminated before convergence is met. By setting Stats to on, the simulation results are displayed, for example, the number of ODE calls and the number of BC calls.

The function bvp $4 \mathrm{c}$ requires an initial guess for the trajectories, for which a constant initialization of $5 \times$ eps is selected for this example using the function bvpinit. An initial guess of zero is not permitted because the BCs would be automatically satisfied and bvp $4 \mathrm{c}$ would fail to run.

In line $8, b v p 4 c$ is called. The four arguments to bvp $4 \mathrm{c}$ are the ODE function $z$ dot $(t, z, w)$ given in Appendix A, which represents (13); the BC function (14), given in Appendix A by the function bcfun $(z 0, z f, z 0, p f)$; the initial guess for the solution solinit; and the previously defined options. The output sol of bvp4c must then be evaluated using the command $z=\operatorname{deval}(\operatorname{sol}, t)$ for the time series $t$. Finally, the state and costate histories may be extracted from $z$.

\subsection{Optimized semi-active control trajectories}

The proposed method is now applied to the previously described TMD model under two loading scenarios. First, a pulse-like disturbance is used to validate that the optimized trajectories satisfy the necessary conditions. Then, optimal semi-active trajectories are computed for a recorded earthquake ground motion. In both cases, a comparison is made between the optimal semi-active controller, the optimized passive TMD, the clipped LQR controller, and the uncontrolled system.

3.5.1. Pulse-like ground motion. The converged optimal semi-active trajectory, the passive trajectory, the clipped LQR trajectory, and uncontrolled trajectory are given in Figure 4, along with the primary structure total acceleration $\left(\ddot{x}_{\mathrm{g}}+\ddot{x}_{\mathrm{s}}\right)$ history and the cumulative objective function $J$. The control force $u(t)$ versus the velocity across the actuator $v(t)$ shows that the semi-active constraint is strictly satisfied.
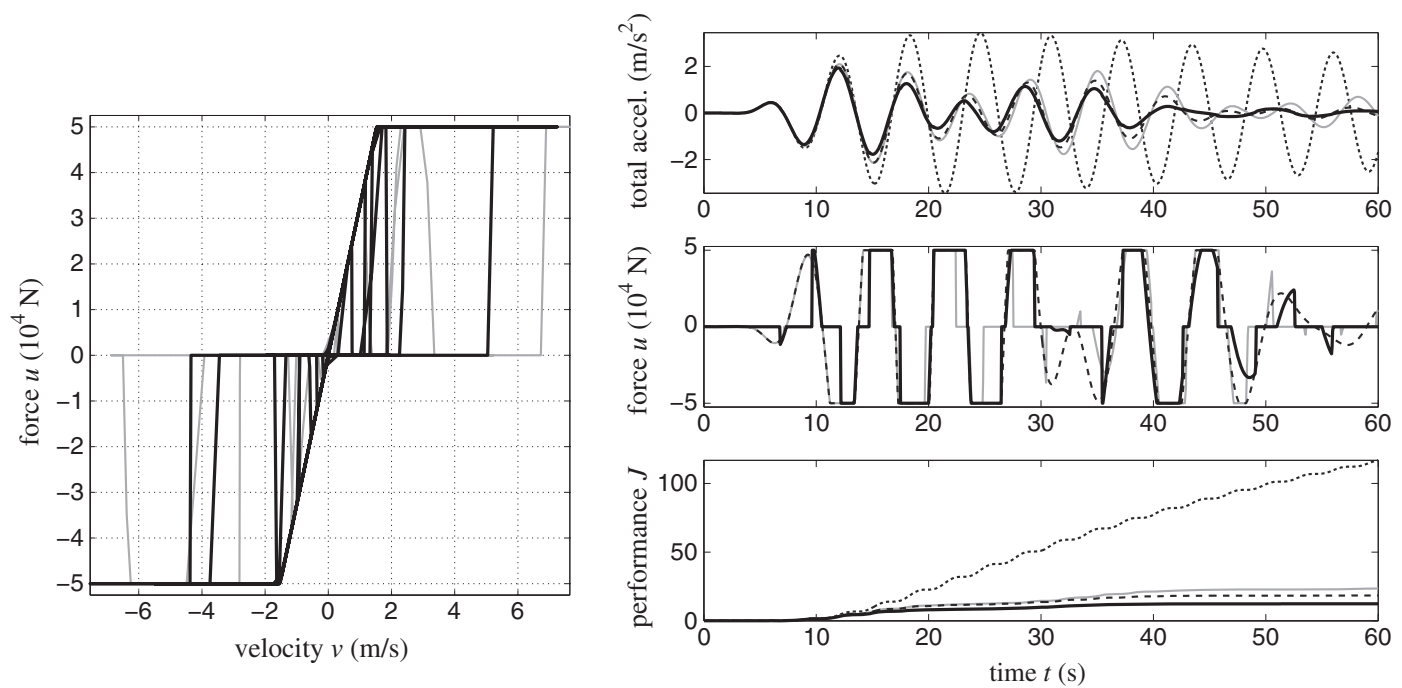

Figure 4. Responses to a pulse-like disturbance: comparison of optimal (thick) with passive (dashed), clipped linear-quadratic regulator (gray), and uncontrolled (dotted). 
As evident from the control force history, the optimal trajectory requires less energy than the clipped LQR and passive controllers (in an $L_{1}$ sense). As observed from Figure 4, the optimal control significantly reduces the mean-square acceleration (i.e., $J$ ), as compared with the passive, clipped LQR, and uncontrolled systems-approximately 33, 47, and 89\%, respectively.

Figure 5 shows the constraint time histories and the corresponding Lagrange multiplier. We see that the complementary slackness condition

$$
\lambda_{i}(t) \begin{cases}=0: & g_{i}(\boldsymbol{x}, u ; t)<0 \\ \geq 0: & g_{i}(\boldsymbol{x}, u ; t)=0\end{cases}
$$

is strictly satisfied by the optimal trajectory. That is to say, the Lagrange multiplier is turned on when the control input desires to be infeasible, pinning the trajectory to the associated constraint boundary. It is clear that the term $\lambda^{\top}(t) \boldsymbol{g}(\boldsymbol{x}, u ; t) \equiv 0, \forall t$.

Figure 6 shows converged control histories from three initial guesses: constant at $5 *$ eps ${ }^{*}$ ones $\left(2^{*} n, 1\right)$, constant at $10^{*}$ ones $\left(2^{*} n, 1\right)$, and the active solution. The number of ODE calls to reach convergence varies: approximately $5.4 \times 10^{5}, 3.6 \times 10^{5}$, and $5.5 \times 10^{5} \mathrm{ODE}$ calls, respectively. The speed of convergence is dependent on the initial guess; with a bad initial guess convergence may be very slow. Nonetheless, for all three initial guesses, the method converges to the same optimal trajectories.

3.5.2. Earthquake ground motion. Now, we consider a recorded earthquake ground motion. The recorded ground motion is the E06230 component of the 1979 Imperial Valley earthquake [25]. Figure 3(b) illustrates the disturbance record. The optimal control force trajectory is illustrated in Figure 7, along with the structure's total acceleration and the performance history. Once again, the optimal control significantly outperforms the uncontrolled system (68\%) and marginally outperforms the passive and clipped LQR controllers (15 and 21\%, respectively). The proposed method is robust enough to handle non-smooth ground motions such as recorded earthquake records. However, convergence required approximately $1.4 \times 10^{6}$ ODE evaluations.
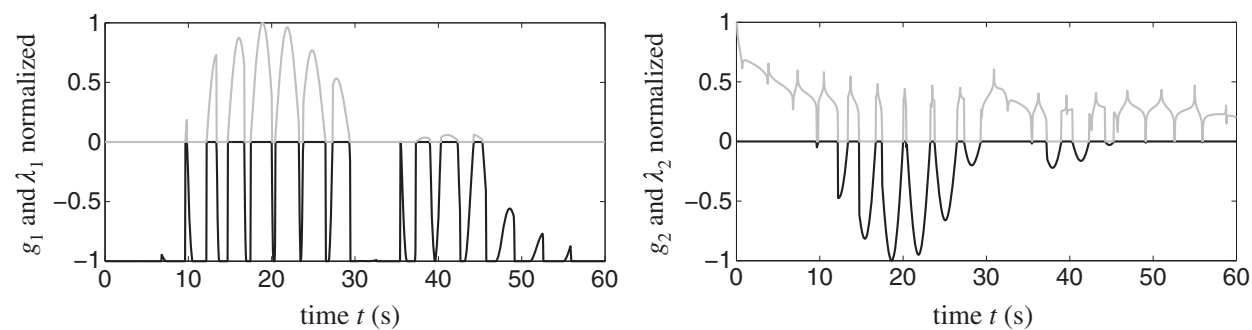

Figure 5. Normalized constraint $g_{i}(\boldsymbol{x}, u ; t)$ and normalized Lagrange multiplier $\lambda_{i}(t)$ time histories (black: constraint $g_{i}(\boldsymbol{x}, u ; t)$; gray: Lagrange multiplier $\left.\lambda_{i}(t)\right)$.

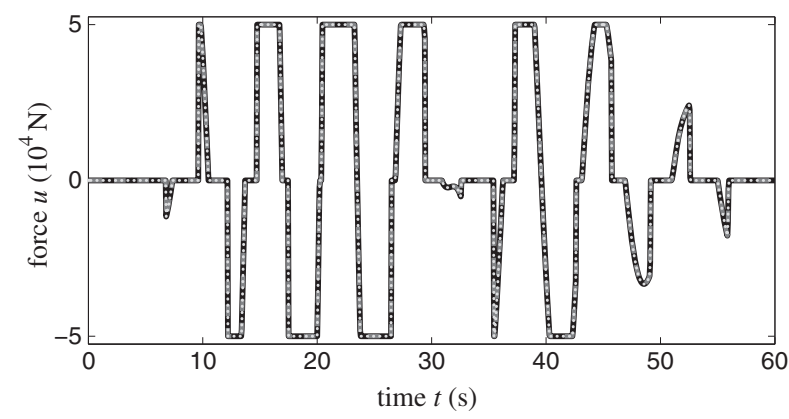

Figure 6. Converged control force histories from three initial guesses (black: $5 \times$ eps; dashed dark gray: 10; dotted light gray: active solution). 

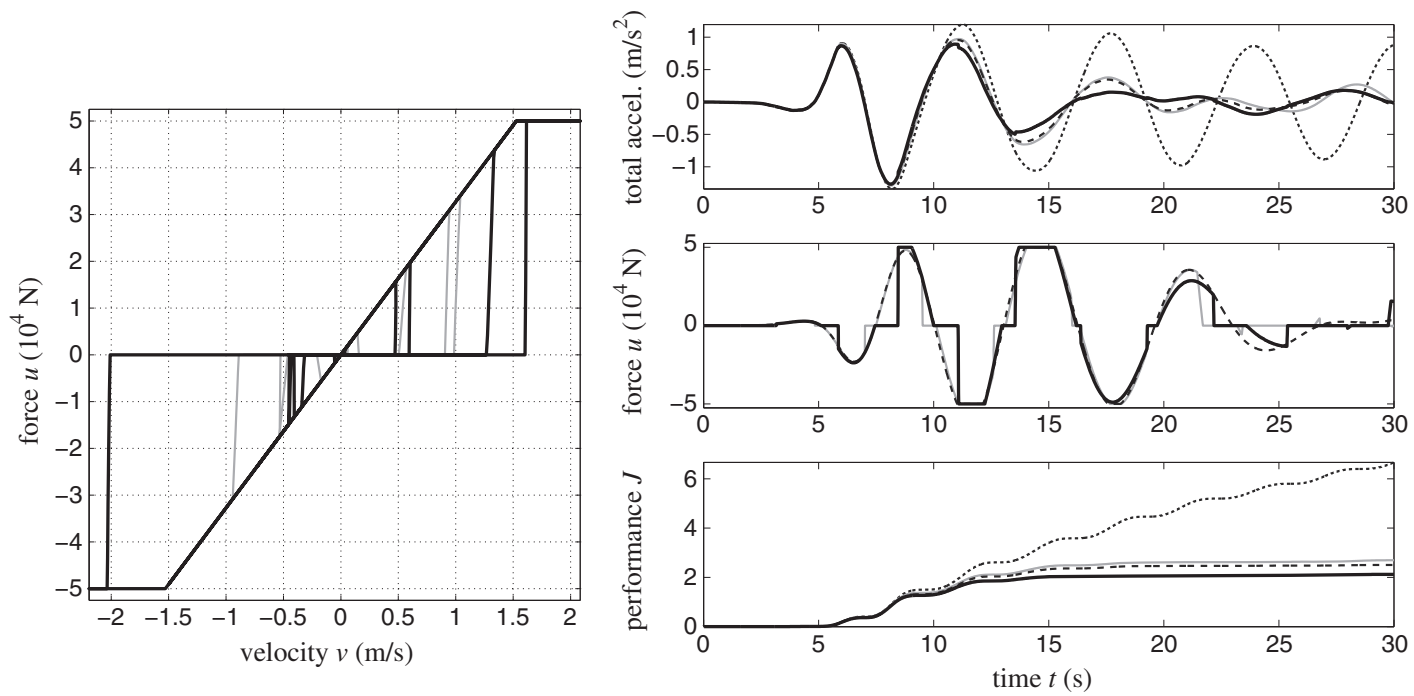

Figure 7. Responses to a recorded earthquake ground motion: comparison of optimal (thick) with passive (dashed), clipped linear-quadratic regulator (gray), and uncontrolled (dotted).

\section{CONCLUSIONS}

The ability to answer the question 'How much could semi-active control improve performance in this application?' can be powerful in establishing the potential for a new semi-active control device or a new semi-active control application. Methods of constrained optimal control, as outlined in this short tutorial paper, provide an easy and ready means to generate such answers. The illustrative example presented is meant to serve only as a guide in solving a rigorously-formulated semi-active control optimization problem, and is therefore, intentionally simple.

\section{FUTURE WORK}

As a tutorial, this paper is intended to encourage the application of optimal semi-active control to answer many important questions. The list of topics in the succeeding text are a sample of studies that could be performed with the methods outlined in this paper.

- Time lag in semi-active control systems can certainly affect the best achievable performance. Applying optimal semi-active control analysis to systems with a time lag will show how important the time lag effects can be. Incorporating time lag into the semi-active damper, where the semi-active damping force is given by $\dot{f}(t)=[u(t)-f(t)] / T_{\text {lag, }}$, and $T_{\text {lag }}$ is the time lag, typically $0.02-0.10 \mathrm{~s}$, would add realism to the simulation results. The state equations would remain linear.

- Different semi-active devices have different behavior. By applying optimal semi-active control analysis to systems controlled by different semi-active devices, the relative potential of different devices can be assessed.

- Semi-active control may potentially provide greater performance for linear structural systems than for nonlinear or hysteretic structural systems. Application of optimal semi-active control analysis to structures with different nonlinearities would show how semi-active control would benefit the behavior of one type of structure as compared with another.

- This study addresses a system with a single semi-active device. Extension to systems with multiple semi-active devices may be studied. In doing so, care must be taken in the saturation function so that coupling between devices is properly accounted for.

- The constraints imposed upon the optimization in this study pertain only to the semi-active device. The constrained optimization methods described in this paper can also be applied to constrain peak responses. For example, in earthquake engineering, peak responses are typically 
of greater interest than mean squared responses [25]. Extending this method to suppress peak response is a matter of removing the quadratic state cost, so that

$$
J=\int_{0}^{t_{\mathrm{f}}} \frac{1}{2} R u^{2}(t) d t
$$

and adding a constraint on the peak response, for example,

$$
g_{x}=\max _{t}\left|x_{\mathrm{s}}(t)\right|-x_{\text {allow }} \leq 0 .
$$

The method here would be to iteratively reduce $x_{\text {allow }}$ until no feasible solution can be found.

- The methodology presented in this paper can be extended to minimizing peak responses by changing the quadratic integrand to a fourth or higher (even) order and by reducing the time horizon to the first few large cycles of response. Doing so would result in nonlinear costate dynamics.

- Optimal control trajectories could be investigated and parameterized in order to develop a class of nonlinear feedback control rules inspired by these optimal performance studies.

\section{Appendix A: MATLAB functions}

The full MATLAB code used to generate the figures in this paper may be found at http://www.duke.edu/ $\sim$ hpgavin/osc. The essentials are given in the succeeding text.

Sample code to run bvp $4 \mathrm{c} \cdot \mathrm{m}$ :

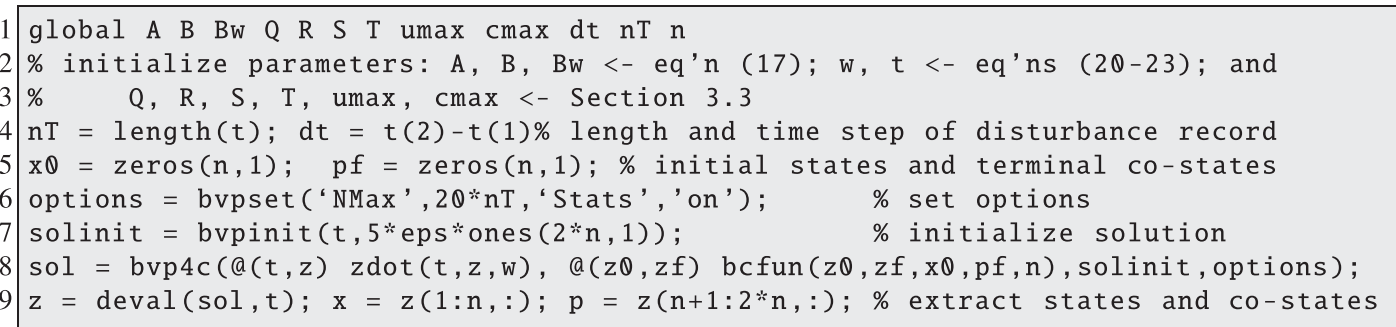

Unconstrained boundary value problem function:

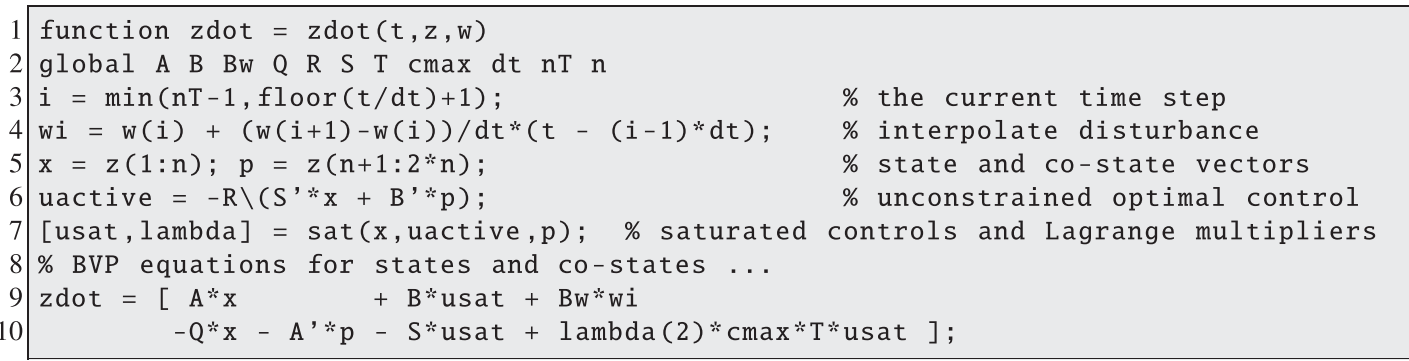

Boundary condition function:

1 function $b c=b c f u n(z \theta, z f, x \theta, p f, n)$

$2 b c=[z \theta(1: n, 1)-x \theta ; z f(n+1: 2 * n$, end $)-p f]$; 
Saturation function:

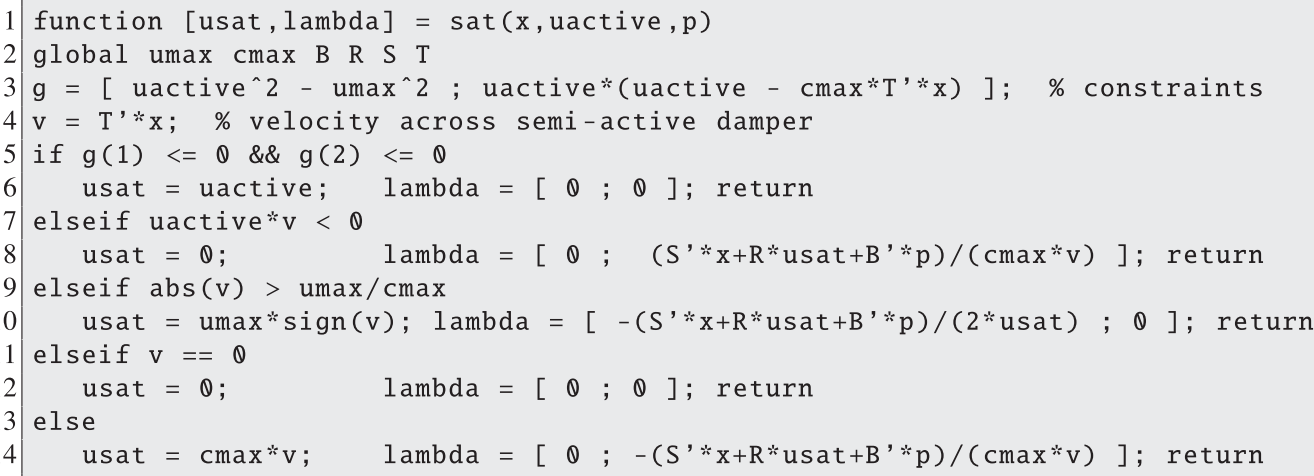

\section{ACKNOWLEDGEMENTS}

This material is based upon the work supported by the National Science Foundation under grant No. NSF-CMMI0900324. Any opinions, findings, and conclusions or recommendations expressed in this material are those of the authors and do not necessarily reflect the views of the National Science Foundation.

\section{REFERENCES}

1. Housner GW, Bergman LA, Caughey TK, Chassiakos AG, Claus RO, Masri SF, Skelton RE, Soong TT, Spencer BF, Yao JTP. Structural control: past, present, and future. Journal of Engineering Mechanics 1997; 123:897-971.

2. Symans MD, Constantinou MC. Semi-active control systems for seismic protection of structures: a state- of-the-art review. Engineering Structures 1999; 21:469-487.

3. Nagarajaiah S. Adaptive passive, semiactive, smart tuned mass dampers: identification and control using empirical mode decomposition, Hilbert transform, and short-term Fourier transform. Structural Control and Health Monitoring 2009; 16:800-841.

4. Kang J, Kim HS, Lee DG. Mitigation of wind response of a tall building using semi-active tuned mass dampers. Structural Design of Tall and Special Buildings 2011; 20:552-565.

5. Ledezma-Ramirez DF, Ferguson NS, Brennan MJ. Shock isolation using an isolator with switchable stiffness. Journal of Sound and Vibration 2011; 330:868-882. doi:10.1016/j.jsv.2010.09.016.

6. Pasala DTR, Nagarajaiah S, Grigoriadis KM. Tracking control of variable stiffness hysteretic-systems using linearparameter-varying gain-scheduled controller. Smart Structures and Systems 2012; 9:373-392.

7. Rodgers GW, Chase JG, Roland T, MacRae GA. Spectral analysis for a semi-active-passive net-zero base-shear design concept. Earthquake Engineering and Structural Dynamics 2012; 41:1207-1216.

8. Cassidy IL, Scruggs JT, Behrens S, Gavin HP. Design and experimental characterization of an electromagnetic transducer for large-scale vibratory energy harvesting applications. Journal of Intelligent Material Systems and Structures 2011; 22:2009-2024.

9. Chen C, Liao WH. A self-sensing magnetorheological damper with power generation. Smart Materials and Structures 2012 21:025 041.

10. Jiang Z, Christenson RE. A fully dynamic magneto-rheological fluid damper model. Smart Materials and Structures 2012 ; 21:065 002 .

11. Laflamme S, Taylor D, Maane MA, Connor JJ. Modified friction device for control of large-scale systems. Structural Control and Health Monitoring 2012; 19:548-564.

12. Lin GL, Lin CC, Lu LY, Ho YB. Experimental verification of seismic vibration control using a semi-active friction tuned mass damper. Earthquake Engineering and Structural Dynamics 2012; 41:813-830.

13. Kirk DE. Optimal Control Theory, An Introduction. Prentice Hall: Mineola, New York, 1970.

14. Tseng HE, Hedrick JK. Semi-active control laws optimal and sub-optimal. Vehicle System Dynamics 1994; 23:545-569.

15. Hrovat D, Margolis DL, Hubbard M. An approach toward the optimal semi-active suspension. Journal of Dynamic Systems, Measurement, and Control 1988; 110:288-296.

16. Leavitt J, Jabbari F, Bobrow JE. Optimal performance of variable stiffness devices for structural control. Journal of Dynamic Systems, Measurement, and Control 2007; 129:171-177.

17. Harvey Jr PS, Gavin HP, Scruggs JT. Optimal performance of constrained control systems. Journal of Smart Materials and Structures 2012; 21:085 001. doi: 10.1088/0964-1726/21/8/085001

18. García CE, Prett DM, Morari M. Model predictive control: theory and practice-a survey. Automatica 1989; 25:335-248.

19. Giorgetti N, Bemporad A, Tseng HE, Hrovat D. Hybrid model predictive control application towards optimal semi-active suspension. International Journal of Control 2006; 79:521-533.

20. MATLAB. version 7.13.0. Natick. The Mathworks Inc.: Massachusetts, USA, 2011.

21. Kierzenka J, Shampine LF. A BVP solver based on residual control and the MATLAB PSE. ACM Transactions on Mathematical Software 2001; 27(3):299-316. doi:10.1145/502800.502801. 
22. Hrovat D, Barak F, Rabins M. Semi-active versus passive or active tuned mass dampers for structural control. Journal of Engineering Mechanics 1983; 109(9):691-705.

23. Den Hartog JP. Mechanical Vibration. Dover: Mineola, New York, 1985.

24. He WL, Agrawal AK. Analytical model of ground motion pulses for the design and assessment of seismic protective systems. Journal of Structural Engineering 2008; 134:1177-1188.

25. Applied Technology Council. Quantification of Building Seismic Performance Factors. (Available from: https://www. atcouncil.org/Projects/atc-63-project.html) [1 September 2012]. 